\title{
Aumento del umbral tusígeno en sujetos sanos con el uso de Levodropropizina
}

\author{
PATRICIA SCHÖNFFELDT G.*, JUAN CÉSPEDES G.*, \\ RICARDO SEPÚLVEDA M.* y M. EUGENIA SALAMANCA H.*
}

\section{INCREASE OF THE COUGH THRESHOLD TO CAPSAICIN IN HEALTHY ADULTS BY ADMINISTERING LEVODROPROPIZINE}

A randomized, prospective, double blind and placebo controlled study was designed to evaluate in 20 non smoker healthy adult volunteers the reproducibility and modification of cough threshold (CT) induced by capsaicin after placebo and Levodropropizine (a new synthetic drug). Adult volonteers of both sexes, mean age 34.9 years old (range: 18-57 years), inhaled increasing concentrations of capsaicin to determine the basal CT: log concentration of capsaicin that induced at least two consecutive coughs. The basal CT was $2.240 \mu M( \pm 0.060$ SE), without differences by sex or age ( $p: n s)$, In 11 out of 18 subjects the CT increased from $2.358( \pm 0,044$ SE) on placebo to $2.469 \mu M( \pm 0.057)$ after Levodropropizine $(p=0.01)$. Two subjects were excluded due to intercurrent disease, not related to the study. No significant adverse reactions were reported during the study. Conclusion: Capsaicin induced reproducible controlled cough in 20 healthy volunteers and Levodropropizine given orally increased the cough threshold.

Key words: Capsaicin; cough threshold; levodropropizine.

\section{RESUMEN}

Se diseñó un estudio aleatorio prospectivo, doble ciego cruzado contra placebo, para estudiar la reproducibilidad y las modificaciones del umbral tusígeno (UT) inducido por capsaicina, luego de administrar placebo y Levodropropizina (un nuevo antitusivo sintético). Se determinó el UT basal (logaritmo de la concentración de capsaicina que induce al menos dos toses consecutivas) en 20 voluntarios adultos sanos, no fumadores de ambos sexos con edad promedio de 34,9 años (rango: 18 a 57 años). El promedio del UT basal fue 2,240 $\mu M( \pm 0,060 \mathrm{ES})$, sin haber diferencias significativas por sexo o edad (p: ns). En 11 de 18 sujetos el UT aumentó de 2,358 $\mu \mathrm{M}( \pm 0,044 \mathrm{ES})$ con placebo a 2,469 $\mu \mathrm{M}( \pm 0,057 \mathrm{ES})$ con Levodropropizina $(p=0,01)$. Dos sujetos fueron excluidos por presentar una enfermedad intercurrente no relacionada con el estudio. No hubo efectos adversos significativos. Conclusión: La capsaicina indujo reproduciblemente el reflejo tusígeno en 20 voluntarios sanos, y la Levodropropizina administrada por vía oral aumentó el umbral tusígeno.

Palabras clave: Tos, capsaicina; umbral tusígeno; Levodropropizina.

* Laboratorio de Función Pulmonar, Instituto Nacional del Tórax. 


\section{INTRODUCCIÓN}

La tos es un eficiente reflejo defensivo del aparato respiratorio, cuyo origen puede estar en diversos territorios, tanto extra como intrapulmonares, principalmente en la vía aérea central y su propósito es la eliminación de secreciones o elementos extraños del pulmón ${ }^{1}$. Sin embargo, la exacerbación o su mantención en el tiempo puede constituirse en un problema per se, ya sea por la frecuencia o la intensidad de ella. Es una causa frecuente de consulta, dada la alteración de la calidad de vida y especialmente del sueño que provoca, recibiendo tratamiento etiológico o mayoritariamente sintomático ${ }^{2,3}$. Una manera de inducir este reflejo y así estudiar su fisiología y posibilidad de modificación terapéutica, está dada por la inhalación de diferentes sustancias, como agua destilada, partículas, sustancias broncoconstrictoras tales como Histamina o Metacolina y sustancias irritantes como ácido Cítrico o Capsaicina. Esta última, extracto de pimienta picante de la familia de los vaniloides, administrada en forma de aerosol de dosis controlada, ha sido estudiada tanto en sujetos sanos como enfermos respiratorios; en algunas publicaciones $^{4,5}$ se muestra que existen diferencias en el UT según la edad y el género de los pacientes.

El umbral tusígeno UT es la concentración de una sustancia, como la Capsaicina, que al ser administrada por aerosol es capaz de provocar a lo menos 2 toses consecutivas en los individuos estudiados y suele expresarse en base logarítmica; existe limitada información de este umbral en sujetos sanos y si bien existen tablas españolas ${ }^{6}$, no hay datos nacionales conocidos. La información disponible señala que la estimulación de tos con Capsaicina, no provoca reacciones adversas significativas ni broncoconstricción?

Con el fin de establecer la técnica de estudio del UT con Capsaicina y su modificación farmacológica, se decidió realizar un estudio prospectivo, aleatorio y doble ciego cruzado controlado por placebo en voluntarios sanos no fumadores, con la medición de la concentración basal y después de la ingesta de un antitusivo. La Levodropropizina es un fármaco antitusígeno sintético, no narcótico, que actuaría inhibiendo las vías aferentes que regulan la generación del reflejo tusígeno ${ }^{8}$ y se encuentra en nuestra farmacopea desde el año 2000 al ser aprobado por el Instituto de Salud Pública, con una dosis sugerida de $60 \mathrm{mg}(10 \mathrm{ml}) 3$ veces al día. Sin embargo, siendo un nuevo fármaco, conocido desde 1988, hay pocos estudios que lo relacio- nan con el UT en la literatura internacional y ninguna en la nacional.

\section{SUJETOS Y MÉTODOS}

Ingresaron 20 voluntarios sanos, que firmaron el consentimiento informado, aprobado por el Comité de Ética del Servicio de Salud Metropolitano Oriente.

\section{Criterios de inclusión}

- Pacientes de ambos sexos.

- Edad entre 18 y 60 años.

- Sin antecedentes de enfermedades crónicas.

- Sin cuadros respiratorios de ningún tipo los últimos 30 días.

- Sin antecedentes de tabaquismo.

- Tener estudio espirométrico normal.

\section{Criterios de exclusión}

- Embarazo y lactancia.

- Analfabetismo.

- No poder cumplir con los controles.

- Antecedente de cefaleas crónicas o jaquecas.

- Antecedente de síntomas alérgicos en los últimos 30 días.

- Uso actual de fármacos sedantes o relajantes o de antihistamínicos.

- Uso crónico de otros medicamentos que interfieran con la tos, v.gr. analgésicos que contengan codeína.

Luego de la selección e inclusión en el estudio de los voluntarios, éstos fueron distribuidos al azar en las diferentes secuencias de medicamentos.

Fármaco y placebo se encontraban en dos frascos idénticos, señalados aleatoriamente como A o B y numerados en forma correlativa (ejemplo 1A - 1B).

Un profesional independiente al estudio preparó los frascos, con similares excipientes. La clave del contenido de cada frasco se guardó, conociéndose ésta sólo al final del estudio.

Las nebulizaciones se realizaron con un nebulizador Hudson, con un flujo de oxígeno de 6 litros por minuto y $3 \mathrm{ml}$ de solución en suero fisiológico cada 4 minutos; las concentraciones de Capsaicina fueron en dosis crecientes desde $62,5(\log 1,796)$ a $500 \mu \mathrm{M}(\log 2,699)$ en progresión de $62,5 \mu \mathrm{M}$ cada vez, hasta determinar la concentración que inducía al menos dos toses consecutivas (UTlog2).

Se determinó la respuesta basal (UTlog2 basal) frente a la estimulación tusígena con Capsaicina 
de cada sujeto, luego se le entregó uno de los frascos numerados del que debía ingerir $10 \mathrm{ml}$ 3 veces al día.

Después de 3 días de consumo y en el plazo de menos de 8 horas de la última dosis, el sujeto fue evaluado en el laboratorio y de no existir factores intercurrentes que impidieran continuar, se repetió la medición del UT frente a nebulización de Capsaicina, consignando el valor obtenido. Luego de tres días sin medicación (período de lavado de droga), tomó de igual forma el segundo frasco de jarabe. Después del tercer día de ingesta y con menos de 8 horas de la última dosis, volvió a repetir la medición del UT.

Todas las mediciones se efectuaron en similar horario.

\section{Criterio de efecto positivo}

De acuerdo a la literatura disponible, se consideró como efecto positivo el aumento de al menos dos concentraciones de Capsaicina necesaria para inducir 2 toses consecutivas, en relación a la concentración basal.

\section{Estudio estadístico}

Se comparó el porcentaje de casos considerados positivos tanto para la droga como para el placebo y se comparó la significación estadística entre la concentración obtenida con la administración de la droga (Drogalog2) y el placebo (Placebolog2) y entre ellos con el basal (UTlog2), mediante el t-test de Student para muestras pareadas. Se comparó también la posible correlación ( $\mathrm{r}$ de Pearson) entre edad y UT y se buscó la existencia de diferente UT entre sexos con ttest para muestras no pareadas. Del mismo modo se buscó la similitud o diferencias del UT entre los sexos respecto a la edad. Se utilizó el sistema estadístico computarizado Epistat Statistical Package y se consideró como significativo toda diferencia de $\mathrm{p}<0,05$.

\section{Visitas}

Los participantes realizaron tres visitas de acuerdo al diseño siguiente:

\section{Visita 1}

Durante esta visita se realizó anamnesis, examen físico, evaluación de la función pulmonar con espirometría y se estudió su UT. El voluntariado fue instruido para usar:

- La medicación del estudio correctamente.

- Un diario en el cual registrara los síntomas que pudiera tener y la toma de medicación durante todo el estudio.

\section{Visita 2}

En esta visita, se le pidió describir cualquier cambio de salud que hubiese experimentado $\mathrm{y}$ se revisó el diario de registro de medicación según pauta previa.

Se estudió el nuevo UT.

- Fue instruido en como usar la medicación del estudio.

- Recibió nuevamente su diario y medicación del estudio.

- Confirmó su última visita.

\section{Visita 3}

Se le pidió que describiese cualquier cambio de salud que hubiese experimentado y se le revisó el diario de registro de medicación.

Se le estudió el nuevo UT.

Se dio por terminada la participación del voluntario en el estudio.

\section{RESULTADOS}

De los 20 sujetos incluidos en el protocolo, 18 lo completaron sin problemas. Dos sujetos completaron sólo la primera fase, siendo excluidos de la evaluación final por haber presentado un cuadro de patología aguda no relacionada con la droga en estudio (resfrío y metrorragia respectivamente), que les impidió realizar la segunda fase. Al finalizar el estudio y abrirse las claves de los frascos utilizados, se observó que uno de estos sujetos había usado la droga y el otro el placebo, con aumento del umbral tusígeno en el primer caso y disminución en el segundo (Tabla 1).

La edad promedio del grupo de 20 sujetos, incluidos inicialmente, fue de 34,9 años (rango 18 a 57), con 11 mujeres (55\%) y no hubo diferencia estadísticamente significativa (prueba de Student) entre la edad promedio de ambos grupos: $34,6( \pm 11,40 \mathrm{DS})$ y $35,1( \pm 9,3 \mathrm{DS})$ años.

La concentración promedio para el umbral tusígeno (UTlog2) de los 20 sujetos que iniciaron el protocolo fue de $2,241 \mu \mathrm{M}(\mathrm{ES} \mathrm{0,071)} \mathrm{en}$ los hombres y de 2.367 (ES 0,069) para las mujeres, sin diferencia significativa entre ellos. La concentración promedio de todo el grupo fue de 2,240 $\mu \mathrm{M}$ (ES 0,060) (Figura 1).

No hubo correlación entre la edad de todo el grupo de sujetos estudiados y el umbral tusígeno ( $\mathrm{r}=0,005 ; \mathrm{p}: \mathrm{ns}$ ), como tampoco la hubo al hacer el estudio separando a los pacientes por sexo.

Al considerar como efecto positivo de la droga el aumento del UT a lo menos en dos concentraciones de capsaicina, 11 de ellos $(61,1 \%)$ 
Tabla 1. Datos generales y umbral tusígeno basal, con levodropropizina y placebo en 20 adultos sanos, no fumadores

\begin{tabular}{|c|c|c|c|c|c|}
\hline Sujeto & Sexo & Edad & UT basal & UT Levod. & UT placebo \\
\hline 1 & $\mathrm{H}$ & 23 & 2,574 & 2,641 & 2,574 \\
\hline 2 & M & 41 & 1,796 & 2,273 & 2,641 \\
\hline 3 & M & 43 & 2,087 & - & 1,796 \\
\hline 4 & $\mathrm{H}$ & 29 & 1,796 & 1,796 & 1,796 \\
\hline 5 & $\mathrm{H}$ & 28 & 2,097 & 2,398 & - \\
\hline 6 & $\mathrm{H}$ & 34 & 2,097 & 2,398 & 2,273 \\
\hline 7 & $\mathrm{H}$ & 27 & 1,796 & 2,495 & 2,398 \\
\hline 8 & $\mathrm{H}$ & 27 & 2,097 & 2,273 & 2,273 \\
\hline 9 & M & 47 & 2,273 & 2,398 & 2,273 \\
\hline 10 & M & 47 & 2,398 & 2,574 & 2,398 \\
\hline 11 & M & 18 & 2,273 & 2,097 & 2,273 \\
\hline 12 & M & 29 & 2,097 & 2,398 & 2,273 \\
\hline 13 & M & 25 & 2,641 & 2,699 & 2,641 \\
\hline 14 & M & 32 & 2,641 & 2,699 & 2,495 \\
\hline 15 & M & 40 & 2,273 & 2,495 & 2,273 \\
\hline 16 & M & 32 & 2,398 & 2,641 & 2,398 \\
\hline 17 & M & 32 & 2,495 & 2,641 & 2,398 \\
\hline 18 & $\mathrm{H}$ & 37 & 2,097 & 2,641 & 2,273 \\
\hline 19 & $\mathrm{H}$ & 57 & 2,495 & 2,641 & 2,398 \\
\hline 20 & $\mathrm{H}$ & 49 & 2,398 & 2,641 & 2,398 \\
\hline \multicolumn{2}{|c|}{ Promedio } & 34,9 & 2,240 & 2,465 & 2,329 \\
\hline \multicolumn{2}{|l|}{ DS } & 10,01 & 0,268 & 0,235 & 0,224 \\
\hline \multicolumn{2}{|l|}{ ES } & 2,24 & 0,060 & 0,054 & 0,051 \\
\hline
\end{tabular}

H: Hombre; M: Mujer; UT: logaritmo umbral tusígeno; DS: Desviación estándar; ES: Error estándar; Levod: Levodropropizina

lo hicieron (Tabla 1). Sólo 2 sujetos $(11,1 \%)$ lo hicieron con el placebo y con la droga simultáneamente, ninguno con placebo exclusivo; 1 lo disminuyó con la droga y 1 no modificó el UT con ningún producto (Tabla 1). En los 18 que completaron el estudio, el promedio de la concentración tusígena después de haber usado la droga (Drogalog2) fue de 2,469 $\mu \mathrm{M}$ (ES 0,057, varianza 0,058 ) y con el placebo (Placebolog2) fue de 2,358 (ES 0,044, varianza 0,035), diferencia estadísticamente significativa $(\mathrm{p}=0,01)$. La diferencia entre el UT inicial de los 18 sujetos y después del uso de placebo no fue significativa y sí lo fue después de Levodropropizina $(\mathrm{p}<0,001)$ (Figura 2).
En relación a posibles efectos adversos, no hubo reacciones alérgicas, sedantes o sistémicas de importancia en ningún sujeto; uno acusó tos con el uso de ambas sustancias, uno refirió sensación de "pecho apretado" también con ambas sustancias, sin alteración funcional, uno presentó dolor abdominal leve con ambas sustancias, uno presentó diarrea leve sólo el primer día de uso de la droga y uno acusó odinofagia con el placebo sólo en el primer día. Dos sujetos refirieron notar mal sabor con ambos jarabes.

La administración de las diferentes concentraciones de Capsaicina y su aplicación, a través de nebulizaciones no presentó problemas para los sujetos en estudio, ni a los operadores. 

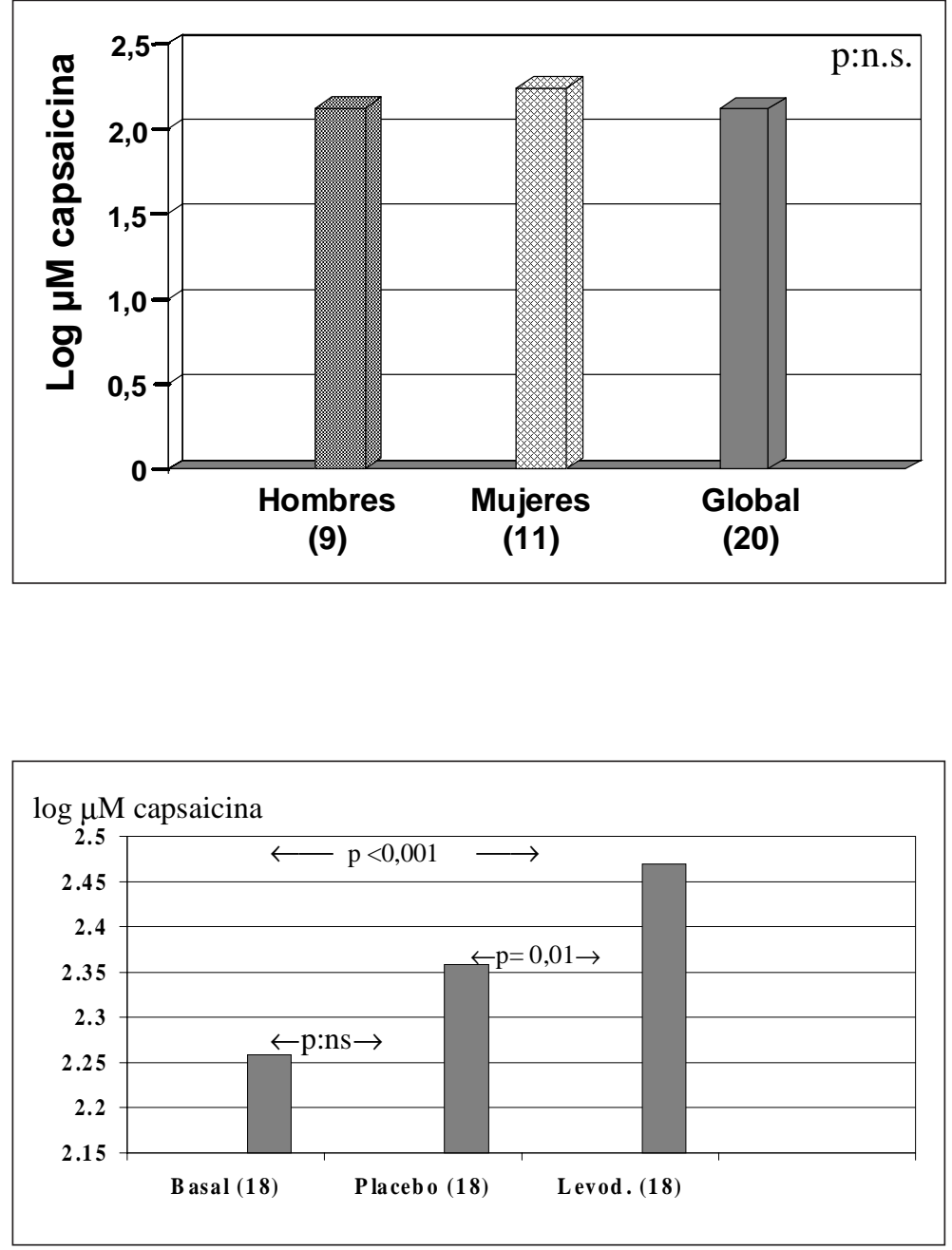

Figura 1. Umbral tusígeno basal en 20 adultos sanos no fumadores. No se observó diferencia significativa entre hombres y mujeres (p: ns prueba de Student para muestras no pareadas). Entre paréntesis se indica el número de sujetos.
Figura 2. Umbral tusígeno basal, con placebo y con Levodropropizina (Levod) en los 18 adultos sanos no fumadores que lograron completar el estudio. El valor de $\mathrm{p}$ fue calculado con la prueba de Student para muestras pareadas.

\section{COMENTARIOS}

A diferencia de lo señalado por Dicpinigaitis et al, en nuestro estudio no encontramos diferencias del UT en relación a la edad y sexo, pudiendo ser lo reducido de la muestra el factor que explique estos resultados. El nivel del UT es relativamente alto, pudiendo incidir que la concentración inicial en nuestro estudio fuese alta, ya que 3 sujetos lo alcanzaron con la primera dosis y tal vez su umbral fuese más bajo y aquellos 4 con los niveles más altos trabajan habitualmente expuestos a inhalar sustancias irritantes (lavan material de laboratorio), factores que deberían considerarse en otro estudio.

El sistema empleado para la determinación del UT fue adecuado y no presentó problemas en su realización ni en su evaluación, siendo tal vez posible de mejorar si además se empleara el estudio de la concentración que provoca 5 toses (log5) en forma simultánea, lo que permitiría quizás ser más específico al tomar ambas concentraciones como factor de cambio a evaluar, lo que debiera estudiarse en futuros estudios.

Si bien era esperable que la droga manifestara su efecto antitusivo, se destaca el alto porcentaje alcanzado, lo que podría ser mayor con el criterio de alza de al menos una concentración para considerarlo positivo, ya que serían 16 de 18 los casos positivos $(88,8)$, pero el placebo también subiría a 6 sujetos, con $33,3 \%$, por lo que parece adecuado considerar que el criterio adoptado para definir el efecto positivo fue correcto en este estudio. 
La diferencia significativa entre Levodropropizina y placebo y lo significativo del aumento del UT con la droga respecto al basal confirman su efectividad como antitusivo.

\section{CONCLUSIÓN}

De los resultados obtenidos en esta muestra podemos concluir que la determinación del umbral tusígeno con Capsaicina inhalada, es un buen método para el estudio de la tos y que la Levodropropizina es una droga segura y efectiva en lograr un aumento de dicho umbral, significativamente superior al placebo.

\section{AGRADECIMIENTOS}

Los autores agradecen al Instituto Farmacéutico Labomed S.A. por su apoyo y auspicio para la realización de este estudio y declaran no tener conflictos de intereses.

\section{BIBLIOGRAFÍA}

1.- IRWIN R S, FRENCH C L, CURLEY F J, ZAWACKI J K, BENETT F M. Managing cough as a defense mechanism and as a symptom. Chest 114 Supplement 1998.

2.- IRWIN R S. Review Appropiate use of antitussives and protussions. Drugs 1993; 46: 80-91.

3.- IRWIN R S. The treatment of cough. Chest 1991; 99: 1477-84.

4.- DICPINIGAITIS P V. Cough reflex sensitivity in cigarette smokers. Chest 2003; 123: 685-8.

5.- DICPINIGAITIS P V, ALLUSON V R, BALDANTI A, NALAMATI J R. Ethnic and gender differences in cough reflex sensitivity Eur Respir J 1992; 5: 296-300.

6.- NIETO CABRERA M L, DE DIEGO DAMIA A, PERPIÑA TORDERA M, MARTÍNEZ PÉREZ E, COMPTE TORRERO L, MACIAN GISBERT V. Cough inducing capsaicin challege test in healthy population. Arch Bronconeumol 2001; 37: 292-6.

7.- ROBERTS M L, DOHERTY M J, MISTER R, PEARSON M G, CALVERLEY P M. Capsaicin responsiveness and cough in asthma and cronic obstructive pulmonary disease. Thorax 2000; 55: 643-9.

8.- MALANDRINO S, MELILLO G, BESTETTI A, BORSA M, GIULIANI P, TONON G. Antitussive propeties of Levodropropizine. Drug 1988; 38:11413. 